\title{
Teleconsulta no Contexto da Covid-19: Experiência de uma Equipe em Cuidados Paliativos
}

\author{
Teleconsultation in the Context of the Covid-19: Experience of the Palliative Care Team
}

Andrea Augusta Castro'(D), Ana Cláudia Chazan' ${ }^{(\mathbb{D})}$, Caroline Peixoto dos Santos'(D), Elza Maria Bayer Candal' ${ }^{\mathbb{D}}$, Luiz Fernando Chazan' ${ }^{\mathbb{D}}$, Patrícia Cristina dos Santos Ferreira! ${ }^{\mathbb{D}}$

\begin{abstract}
RESUMO
Introdução: Cuidados paliativos é um direito que deve ser assegurado. Diante da pandemia causada pela Covid-19, a recomendação de isolamento social foi uma das estratégias para o enfrentamento.

Relato de experiência: A equipe do Núcleo de Cuidados Paliativos do Hospital Universitário Pedro Ernesto organizou o fluxo de atendimento aos pacientes por meio do acréscimo ao atendimento presencial das modalidades de teleconsulta. Essa ampliação gerou um aumento da comunicação do paciente e da família dele com a equipe de saúde, preservando, dessa forma, um plano terapêutico baseado no cuidado, acolhimento e respeito adequado a esse período de enfretamento do novo coronavírus.

Discussão: A comunicação entre os profissionais das diversas áreas de saúde do núcleo se intensificou quanto às discussões dos casos e desfechos apresentados. Apresentam-se as principais estratégias, os processos, as percepções e os desafios desenvolvidos e enfrentados pela equipe interdisciplinar. Destaca-se o papel da equipe de agente facilitador da integração e comunicação entre o paciente, o Hupe e a rede SUS. Conclusão: A equipe do núcleo foi fundamental para que os pacientes e familiares assistidos pudessem atravessar o momento com mais tranquilidade, segurança e cuidado integral.
\end{abstract}

Palavras-chave: Cuidados paliativos; Telemonitoramento; Infecções por Coronavírus; Práticas Interdisciplinares; Pandemia.

\begin{abstract}
Introduction: Palliative care is a right that must be ensured. Faced with the Covid-19 pandemic, social isolation was one of the recommended strategies for tackling the situation.

Experience report: The team from the Palliative Care Unit of Pedro Ernesto University Hospital organized the flow of care for patients by adding teleconsultation to face-to-face care, with the aim of expanding communication between patients and their families and the health team, thus preserving a therapeutic plan based on the care, reception and adequate respect for this period of coping with the new coronavirus.

Discussion: Communication between professionals from the different health areas of the unit has intensified due to discussions of the cases and outcomes presented. This article presents the main strategies, processes, perceptions and challenges developed and faced by the interdisciplinary team. The team plays a prominent role in facilitating patient integration and communication with the HUPE and the SUS network.

Conclusion: The importance of the team is reflected on, in terms of how the center assists patients and family members to help them through the difficult experience with more peace of mind, security and comprehensive care.
\end{abstract}

Keywords: Palliative Care; Telemonitoring; Coronavirus Infections; Interdisciplinary Practices; Pandemic.

'Universidade do Estado do Rio de Janeiro, Rio de Janeiro, Rio de Janeiro, Brasil.

Correspondência:

Andrea Augusta Castro.

Avenida Professor Manoel de Abreu, 444, $2^{\circ}$ andar, Vila Isabel, Rio de Janeiro, RJ, Brasil. CEP: 20550-170.

E-mail: castro.andreaaugusta@gmail.com

Recebido em 10/08/20; Aceito em 26/08/20. 


\section{INTRODUÇÃO}

Cuidados paliativos (CP) é uma modalidade assistencial a ser oferecida a todos que recebem o diagnóstico de uma doença que ameaça a vida, havendo ou não a possibilidade de intervenções curativas. O foco do cuidado é a pessoa, para a qual há muito a oferecer, mesmo quando a doença está ativa e o tempo de vida é limitado ${ }^{1,2}$.

Segundo Gamondi et al. ${ }^{3}$, a prática colaborativa de uma equipe interprofissional é o modelo de atenção almejado para o atendimento das necessidades apresentadas pelos indivíduos em CP e pelas famílias deles. Entre as competências centrais da equipe, são ressaltados a defesa da autonomia, dignidade e qualidade de vida dos pacientes, a comunicação e a construção de vínculos ${ }^{4}$.

Em 2009, foi criado o Núcleo de Cuidados Paliativos (NCP), no processo de acreditação do Hospital Universitário Pedro Ernesto (Hupe) da Universidade do Estado do Rio de Janeiro (Uerj), como um Centro de Assistência de Alta Complexidade em Oncologia (Cacon). Em 2016, a equipe multiprofissional do NCP foi reestruturada, mas vem dando continuidade às ações de atenção, educação, extensão e pesquisa em CP, para difundir e consolidar essa modalidade de cuidado na rede do Sistema Único de Saúde (SUS), considerada um direito humano5.

Em 17 de março de 2020, o governador do estado do Rio de Janeiro decretou o isolamento social como forma de combate a pandemia da coronavirus disease 2019 (Covid-19), e o Hupe tornou-se referência para a internação de pacientes com as formas moderada e grave da doença, sendo necessária a reorganização dos serviços ambulatoriais para oferecer o cuidado aos pacientes de maior risco e, ao mesmo tempo, protegê-los e a equipe de saúde da infecção pelo coronavírus (Sars-CoV-2) ${ }^{6}$.

Pode-se depreender a importância da manutenção do atendimento aos pacientes em CP e aos familiares deles, dada a ampliação da angústia e do medo diante de mais uma ameaça a uma saúde já fragilizada. Nesse sentido, a equipe do NCP/Hupe fez a transição do seu processo de trabalho para incluir a telessaúde (TS) na continuidade do cuidado destinado aos pacientes.

Telessaúde pode ser definida como a prestação de serviços de saúde por profissionais da área, quando a distância é um fator crítico, usando Tecnologias de Informação e Comunicação (TICS) para o intercâmbio de informações válidas para o diagnóstico, tratamento e prevenção das doenças e lesões, para a pesquisa e avaliação, e para a educação continuada dos profissionais de saúde, com o interesse de promover a saúde dos indivíduos e suas comunidades?.
A TS está crescendo e sua aplicação em CP é vista como uma solução para as demandas desses serviços ${ }^{8}$.

Este trabalho tem como objetivos descrever e analisar as potencialidades e desafios da inserção da TS no processo de trabalho da equipe do NCP/Hupe durante o período de março a julho de 2020.

\section{RELATO DA EXPERIÊNCIA}

Neste período de isolamento social, o ponto de partida para facilitar a comunicação da equipe sobre o fluxo de atendimento aos pacientes do NCP foi a criação e o compartilhamento de uma agenda virtual armazenada em uma nuvem (drive) pela equipe e a definição de que a teleconsulta seria ofertada aos pacientes que tivessem tido ao menos uma consulta presencial com a equipe e que optassem a não ir à consulta presencial. Entende-se por teleconsulta o atendimento remoto com profissional especializado de acordo com sua área de formação, por exemplo: telemedicina para médicos, telefonoaudiologia para fonoaudiólogos, telepsicologia para psicologia etc.

A teleconsulta possibilitou o acompanhamento da evolução dos sintomas pelo uso da Escala de Avaliação de Sintomas Edmonton (Edmonton Symptom Assessment System - ESAS) e do prognóstico pela Escala de Performance de Karnofsky (Karnofsky Performance Status Scale - KPS). Inicialmente, realizaram-se ligações telefônicas, e, após algumas semanas e com apoio da TS da Uerj, foi oferecida a plataforma Cisco Webex para videoconferências que apresenta o selo da Health Insurance Portability and Accountability Act (HIPAA) que estabelece um conjunto de regras nacionais para proteção de certas informações de saúde.

Alguns pacientes optaram pelo contato telefônico, dadas as dificuldades de acesso à internet e à tecnologia. Os que optaram pela videoconferência foram orientados pela equipe quanto à instalação do software. Realizou-se um treinamento para o uso, e enviou-se o Termo de Consentimento Livre e Esclarecido para realização do procedimento.

A confirmação das consultas era feita pelas psicólogas da equipe que, além fornecerem suporte emocional aos pacientes, identificavam as necessidades específicas de teleconsulta e as comunicavam aos respectivos profissionais de saúde por WhatsApp e as registravam no drive. O reagendamento era feito de acordo com a complexidade: baixa, média ou alta conforme os indicadores de desempenho e funcionalidade da KPS e o monitoramento de sintomas da ESAS9,10. Após a teleconsulta especializada, os pacientes seguiam, se necessário, em acompanhamento por essas profissionais ou, caso contrário, aguardavam o próximo contato da equipe, na véspera da data do agendamento seguinte. 
Cabe ressaltar que os pacientes que evoluíam com sintomas respiratórios e suspeita de Covid-19 eram encaminhados à triagem específica do Hupe, evitando-se, dessa forma, o contato com a equipe de CP e outros pacientes, medida que foi ao encontro da literatura que corrobora a importância de manter os prestadores de CP livres da Covid-19 dada a ampliação de demandas no período da pandemia para a equipe ${ }^{11}$.

Durante esse período, a equipe se reuniu por webconferências para o aperfeiçoamento dos fluxos e procedimentos acordados em média três vezes por mês, de modo a melhorar a gestão do tempo da consulta presencial e ampliar o acesso de pacientes de "primeira vez". Além disso, a equipe interagiu mais frequentemente por WhatsApp, investiu na educação permanente, organizou uma live sobre $\mathrm{CP}$ e Covid-19 e participou de dois webgrupos de suporte e reflexão sobre o trabalho, coordenados por um psiquiatra psicanalista.

Elegeram-se dois temas a serem abordados nesses grupos: 1. "Luto em tempos de pandemia", que refere-se às experiências vividas pela equipe nos âmbitos profissional e pessoal; e 2. "Potencialidades e desafios do trabalho da equipe durante a pandemia", cujo produto das reflexões é descrito a seguir.

\section{Potencialidades}

- Fortaleceu a identidade de equipe por meio da ampliação dos espaços de reflexão sobre o trabalho e as situações vivenciadas.

- Fortaleceu o vínculo de confiança e afeto entre equipe, pacientes e famílias.

- Melhorou a comunicação, o respeito mútuo e a confiança no trabalho interprofissional, qualificando a atenção aos pacientes e familiares.

- Permitiu o aperfeiçoamento da gestão de casos pela maior integração da equipe com os demais serviços do Hupe e com a rede de atenção primária do SUS.

- Permitiu o desenvolvimento de empatia da equipe, dada a maior aproximação com os contextos familiares e comunitários dos pacientes.

\section{Desafios}

- Estimar necessidade da ampliação da carga horária dos profissionais por causa do acompanhamento contínuo aos pacientes e familiares.

- Administrar mensagens e ligações de familiares em qualquer dia da semana ou horário.

- Lidar com a diminuição de profissionais na equipe, impedidos de trabalhar por estarem com suspeita ou com a Covid-19, por cuidarem de familiares vulneráveis ou por atuarem em outros serviços do Hupe.

- Observar a regulamentação específica nos ambientes remotos visando à garantia da segurança do paciente e dos profissionais.

- Implementar a incorporação de ambientes virtuais institucionais em um novo processo de trabalho.

- Construir a rede de atenção integrada para a transição de cuidados (atenção ou internação domiciliar e hospice), retaguarda hospitalar e serviços de urgência requeridos.

\section{DISCUSSÃO}

O processo de refletir e pensar sobre a experiência relatada mostra-se um desafio, pois o trabalho está em curso e o distanciamento reflexivo torna-se mais difícil.

A pandemia da Covid-19 causou uma grande mudança na distribuição dos cuidados de saúde, tanto no panorama internacional quanto no nacional. A garantia do direito ao acesso à saúde de grupos invisibilizados como o dos pacientes em CP foi confrontada por essa calamidade da saúde pública, mas não poderia ser negligenciada ${ }^{12}$.

A atuação da equipe interdisciplinar ajuda a romper o ciclo dor-medo-mais dor, auxiliando os pacientes e seus familiares a resgatar suas forças, dando oportunidade para falarem de suas dores, de seus receios e das novas perspectivas, e também das dificuldades em lidar com a ameaça da Covid-19, uma doença com baixo nível de conhecimento e de recursos terapêuticos ${ }^{13,14}$.

Os princípios do acompanhamento dos pacientes em CP são o alívio do sofrimento grave e o tratamento de complicações agudas graves do câncer (como compressão medular, metástases cerebrais sintomáticas em pacientes com bom desempenho, dor etc.), gerenciar o controle de sintomas por TS, quando possível, e organizar serviços de atendimento domiciliar, sempre que possível, para pacientes com altas necessidades antecipadas de $C \mathrm{P}^{15}$.

A TS surgiu rapidamente, como uma opção à consulta presencial, para mitigar o risco de exposição viral para pacientes e profissionais, visto que muitos apresentam alto risco de desenvolver morbimortalidade relacionada à Covid-19. Foi proposta como um meio de garantir cuidados continuados, facilitando o distanciamento físico e diminuindo a carga dos cenários da prática clínica. Para outros autores, a TS tornouse uma estratégia integral para fornecer $\mathrm{CP}$ e permitir a continuidade das prescrições e da renovação de opioides ${ }^{16,17}$.

Flexibilidade e resiliência, que podem ser definidas como a "capacidade de se recuperar da adversidade fortalecido e mais resolutivo"18. têm sido fundamentais para superar problemas 
críticos. Dessa forma, o SUS teve que rapidamente se adaptar às novas práticas em saúde. Após a liberação legal dos conselhos, a TS, instrumento corroborado pela literatura nacional, foi implementada por nossa equipe. Por meio da utilização da TS, observamos a melhoria no manejo dos sintomas, conforto no atendimento e satisfação do paciente e da família' ${ }^{19}$.

A TS tem sido uma ferramenta extremamente valiosa, mas, ainda assim, alguns autores apontam possíveis barreiras, como complicações relacionadas à tecnologia na população idosa e frágil ${ }^{20}$ e à presença ou não de familiares. Além disso, os pacientes, assim como os médicos, nem sempre se sentem à vontade com o atendimento médico não presencial ${ }^{21}$. Pelo menos um estudo controlado randomizado de teleconsultas semanais demonstrou, de forma significativa, mais ansiedade e maior angústia no atendimento não presencial por parte dos profissionais de saúde, o que nos leva a pensar se a ampliação da comunicação dos membros da equipe do NCP pode ter sido a forma encontrada de aplacar a angústia deles ${ }^{22}$.

A carga de trabalho da equipe do NCP aumentou durante a pandemia, e ficamos disponíveis aos nossos pacientes por meio das tecnologias de informação e comunicação (TICs). Os processos que antes eram realizados quase exclusivamente de forma presencial foram maximizados para atendimento a distância - revisões regulares, demandas de intercorrências, dúvidas e agendamentos -, ficando o atendimento presencial restrito a casos novos com alto grau de complexidade e quando o paciente assim o desejasse ${ }^{23}$.

Com a pandemia, o cenário dos $\mathrm{CP}$ tornou-se mais complexo. A crise planetária trouxe uma ameaça compartilhada por todos, profissionais, pacientes e famílias. A necessidade de proteger e se proteger da exposição ao coronavírus promoveu uma proximidade afetiva inédita. A carga emocional eleva-se e torna-se necessário ajustarmelhor as necessidades dos pacientes com as possibilidades da equipe de saúde. Possibilidades que estão diretamente relacionadas à resiliência.

Contudo, o aumento da carga de trabalho dos profissionais envolvidos tem resultado no seguinte questionamento:

Por quanto tempo é possível manter esse esquema de cuidado sem uma adequada regulamentação desse novo processo de trabalho?

\section{CONCLUSÃO}

A equipe multidisciplinar do NCP ajustou-se às necessidades impostas pela pandemia para manter 0 cuidado destinado aos pacientes, o que demonstra empatia, flexibilidade e resiliência.

A despeito da redução do contato face a face com os pacientes, a TS permitiu que a equipe captasse pistas e emoções essenciais para uma boa comunicação, desenvolvesse competências clínicas e socioemocionais, e acompanhasse de perto o desfecho da vida dos pacientes.

\section{CONTRIBUIÇÃO DOS AUTORES}

Todos os autores participaram da concepção deste relato de experiência e da análise e discussão dos resultados obtido.

\section{CONFLITO DE INTERESSES}

Os autores declaram não haver conflito de interesses neste relato.

\section{REFERÊNCIAS}

1. Gomes ALZ, Othero MB. Cuidados paliativos. Estud Av. 2016;30(88):155-66.

2. Carroll T, El-Sourady M, Karlekar M, Richeson A. Primary palliative care education programs: review and characterization. Am J Hosp Palliat Care. 2019 June;36(6):546-9.

3. Gamondi C, Larkin P, Payne S. Competências centrais em cuidados paliativos: um guia orientador da EAPC sobre educação em cuidados paliativos - parte 1. Eur J Palliat Care. 2013;20(2):86-91.

4. Peduzzi M, Norman IJ, Germani ACCG, da Silva JAM, Souza GC. Educação interprofissional: formação de profissionais de saúde para o trabalho em equipe com foco nos usuários. Rev Esc Enferm USP. 2013;47(4);977-83.

5. Hennemann-Krause L, Araújo JÁ, Florentino DM, Petersen EM. Cuidados paliativos: o valor da pessoa e sua história no Hupe. Revista HUPE. 2015;14 (supl. 1):19-27.

6. Governo do Estado do Rio de Janeiro. Decreto $n^{\circ} 46.980$, de 19 de março de 2020. Atualiza as medidas de enfrentamento da propagação decorrente do novo Coronavírus (COVID-19) em decorrência da situação de emergência em saúde, e dá outras providências. Diário Oficial do Estado; 19 mar 2020 [acesso em: 9 ago 2020]. Disponível em: https:// www.legisweb.com.br/legislacao/?id=391093.

7. Brasil. Guia metodológico para programas e serviços em telessaúde. Brasília: Ministério da Saúde; 2019 [acesso em 2 set 2020]. Disponível em: http://www.ans.gov.br/images/MS-telessaude-manual_2019.pdf.

8. Hancock S, Preston N, Jones $\mathrm{H}$, Gadoud A. Telehealth in palliative care is being described but not evaluated: a systematic review. BMC Palliat Care. 2019;18(1):114 [acesso em 8 set 2020]. Disponível em: https:// assets.researchsquare.com/files/rs-37535/v1/57e20978-b996-4ee6-80c521829a54f3ad.pdf.

9. Mor V, Lalibert L, Morris JN, Wiemann M. The Karnofsky Performance Status Scale. An examination of its reliability and validity in a research setting. Cancer. 1984;53(9):2002:7.

10. Monteiro DR, Kruse MHL, Almeida MA. Avaliação do instrumento Edmont Symptom em cuidados paliativos: revisão integrativa. Rev Gaúcha Enf. $2010 \mathrm{dez} ; 31(4): 785-93$.

11. Yang $K$, Sheng $Y$, Huang $C$, Jin $Y$, Xiong $N$, Jiang $K$, et al. Clinical characteristics, outcomes, and risk factors for mortality in patients with cancer and COVID-19 in Hubei, China: a multicentre, retrospective, cohort study. Lancet Oncol. (2020);21:904-13.

12. Burlá C, Py L. Cuidados paliativos: ciência e proteção ao fim da vida. Cad Saude Publica. 2014;30(6):1-3.

13. Zhang L, Zhu F, Xie L, Wang C, Wang J, Chen R, et al. Clinical characteristics of COVID-19-infected cancer patients: a retrospective case study in three hospitals within Wuhan, China. Ann Oncol. 2020;31:894-901.

14. Guan WJ, Ni ZY, Hu Y, Liang WH, Ou CQ, He JX, et al. Clinical characteristics of coronavirus disease 2019 in China. N Engl J Med. 2020;382:1708-20.

15. Aapro AAM, Addeo A, Acierto P, Berg T, Betella I, Blay J, et al. ESMO guideline: cancer patient management during the COVID-19 pandemic [acesso em 18 jun 2020]. Disponível em: https://www.esmo.org/ guidelines/cancer-patient-management-during-the-covid-19-pandemic. 
16. Mehta AK, Smith TJ. Palliative care for patients with cancer in the COVID-19 era. JAMA Oncol. Published online May 7, 2020 [acesso em 10 ago 2020]. Disponível em: https://jamanetwork.com/journals/ jamaoncology/fullarticle/2765828.

17. Hoek PD, Schers HJ, Bronkhorst EM, Vissers KCP, Hasselaar JGJ. The effect of weekly specialist palliative care teleconsultations in patients with advanced cancer -a randomized clinical trial. BMC Med. 2017;115:119.

18. Walsh F. Family resilience: a framework for clinical practice. Fam Process. 2003;42(1):1-18 [acesso em 7 ago 2020]. Disponível em: http://www.celf. ucla.edu/2010_conference_articles/Walsh_2003.pdf.

19. Worster B, Swartz K. Telemedicine and palliative care: an increasing role in supportive oncology. Curr Oncol Rep. 2017;19:37.

20. Zhang L, Zhu F, Xie L, Wang C, Wang J, Chen R, et al. Clinical characteristics of COVID-19-infected cancer patients: a retrospective case study in three hospitals within Wuhan, China. Ann Oncol. 2020;31:894-901.
21. Funderskov KF, Danbjorg DB, Jess M, Munk L, Zwisler ADO, Dieperink KB. Telemedicine in specialised palliative care: healthcare professionals' and their perspectives on video consultations-a qualitative study. J Clin Nurs. 2019;28:3966-76.

22. Hoek PD, Schers HJ, Bronkhorst EM, Vissers KCP, Hasselaar JGJ. The effect of weekly specialist palliative care teleconsultations in patients with advanced cancer - a randomized clinical trial. BMC Med. 2017;115:119.

23. Sampaio SGSM, Dias AM, de Freitas R. Orientações do serviço médico de uma unidade de referência em cuidados paliativos oncológicos frente à pandemia de Covid-19. Rev Bras Cancer. 2020;66(TemaAtual):e-1058 [acesso em 8 set 2020]. Disponível em: https://rbc.inca.gov.br/revista/ index.php/revista/article/view/1158. 